\title{
Unperturbed Mean-Square Radius of Gyration of 1,2-Polybutadiene ${ }^{\dagger}$
}

\author{
Haizhu $\mathrm{MA}^{\dagger \dagger}$ and Linxi ZHANG* \\ Department of Mathematics-Physics, Zhejiang Institute of Education, \\ Hangzhou, 310012, People's Republic of China \\ * Department of Physics, Hangzhou University, \\ Hangzhou 310028, People's Republic of China
}

(Received January 19, 1993)

\begin{abstract}
Taking account of the geometric structure of side groups, the unperturbed mean-square radius of gyration $\left\langle S^{2}\right\rangle$ of the typical polymer chains are derivated by using the rotational isomeric state theory. If the masses of the side groups are ignored, the expression of $\left\langle S^{2}\right\rangle$ is in agreement with that reported by Flory. The root-mean-square radius of gyration of isotactic 1,2-polybutadiene is numerical calculated as $\left\langle S^{2}\right\rangle^{1 / 2}=0.298 \mathrm{M}^{1 / 2}$ and the characteristic ratio of mean-square radius of gyration is greater $11 \%$ than that without considering the geometric structure of side groups.
\end{abstract}

KEY WORDS Rotational Isomeric State / Mean-Square Radius of Gyration / Side Groups / Geometric Structure / 1,2-Polybutadiene /

The rotational isomeric state approximation (RIS) has been used successfully to treat several properties of the polymer chains. ${ }^{1,2}$ The theory is widely used to calculate the characteristic ratio $C_{n}=\left\langle R^{2}\right\rangle / N l^{2}$ and the temperature coefficient $\mathrm{d}\left(\ln \left\langle R^{2}\right\rangle\right) / \mathrm{d} T$ of the mean-square end-to-end distance $\left\langle R^{2}\right\rangle$ of polymer chains. In general, $\left\langle R^{2}\right\rangle$ is obtained from the meansquare radius of gyration $\left\langle S^{2}\right\rangle$, measured by light-scattering or small-angle-neutron-scattering. ${ }^{3-7}$ Therefore, it is particularly important to estimate the value of $\left\langle S^{2}\right\rangle$. The meansquare radius of gyration of polymer chains without considering side groups, such as polyethylene (the effect of bond $\mathrm{C}-\mathrm{H}$ is ignored), have been investigated by using the RIS theory. Recently, $\left\langle S^{2}\right\rangle$ for the polymer with single side group has been studied. ${ }^{8-10}$ The calculated values of $\left\langle S^{2}\right\rangle$ for polyethylene (PE) and polypropylene (PP) with considering side groups $(\mathrm{C}-\mathrm{H})$ and $\left(\mathrm{C}-\mathrm{CH}_{3}\right)$ are more approched to the experimental data. In this paper, the mean-square radius of gyration of polymer chains with two side groups, which have the different geometric structures and the heavier group masses, are investigated by using the RIS theory, and the expression for $\left\langle S^{2}\right\rangle$ may be apply to 1,2-polybutadiene (1,2-PBD), $\mathrm{PP}$, polydimethylsiloxane (PDMS), and other monsubstituted polymers.

\section{INVESTIGATION OF $\left\langle S^{2}\right\rangle$ FOR POLYMERS WITH DIFFERENT SIDE GROUPS}

A monosubstituted polymer chain with two side groups, shown in Figure 1(a), consists of $x$ monomeric units. Each contains the atoms $\mathrm{a}, \mathrm{b}, \mathrm{c}$, and $\mathrm{f}$. The masses are $m_{\mathrm{a}}, m_{\mathrm{b}}, m_{\mathrm{c}}$, and $m_{\mathrm{f}}$, and the bond lengths $l_{\mathrm{a}}, l_{\mathrm{b}}, l_{\mathrm{c}}$, and $l_{\mathrm{f}}$, respectively. The skeletal atoms are numbered from 0 to $2 x$, and the side groups $\mathrm{b}$ and $\mathrm{c}$ are

† Project supported by Zhejiang Provincial National Science Foundation of China.

${ }^{\dagger}$ To whom all correspondence should be addressed. 
from $(x+1)$ to $3 x$, and $(3 x+1)$ to $4 x$, respectively. The square radius of gyration of the polymer is defined by

$$
S^{2}=M^{-1} \sum_{j=0}^{4 x} m_{j} s_{j}^{2}
$$

where $M$ is the molecular weight, $m_{j}$ is the mass of atom (or group) $j$, and $s_{j}$ is the distance of atom $j$ from the center of mass of the chain. Equation 1 may be also expressed as ${ }^{1}$

$$
\begin{aligned}
S^{2} & =M^{-2}\left(\sum_{0 \leq i<j \leq 2 x}+\sum_{i=0}^{2 x} \sum_{j=2 x+1}^{4 x}\right. \\
& \left.+\sum_{i=2 x+1}^{3 x} \sum_{j=3 x+1}^{4 x}+\sum_{2 x+1 \leq i<j \leq 4 x}\right) m_{i} m_{j} r_{i j}^{2}
\end{aligned}
$$

where $r_{i j}$ is the distance from atom $i$ to $j, r_{i j}$ can be calculated by following way. The skeletal bond vector $\vec{l}_{j}$ is founded on the bond $j$ with the direction from atom $j-1$ to $j$. Here, vector $\vec{l}_{j}$ is also expressed by matrix $\bar{l}_{j}$

$$
\bar{l}_{j}=\left[\begin{array}{lll}
l_{j} & 0 & 0
\end{array}\right]^{T}
$$

with $3 \times 1$ orders. A series of Cartesian reference frames $\left\{x_{j}, y_{j}, z_{j}\right\}$ affixed to consecutive skeletal bond vectors are connected by the axis transformation matrices $T_{j}$ expressed by

$$
\begin{aligned}
T_{j}= & {\left[\begin{array}{ccc}
\cos \theta & \sin \theta & 0 \\
\sin \theta \cos \Phi & -\cos \theta \cos \Phi & \sin \Phi \\
\sin \theta \sin \Phi & -\cos \theta \sin \Phi & -\cos \Phi
\end{array}\right]_{j} } \\
& =T\left(\theta_{j}, \Phi_{j}\right)
\end{aligned}
$$

where $\theta_{j}$, the supplementary of bond angle, is the angle between bond vectors $\vec{l}_{j}$ and $\vec{l}_{j+1}$. That is, $\theta_{\mathrm{a}}$ or $\theta_{\mathrm{f}}$ is between vectors $\vec{l}_{\mathrm{a}}$ and $\vec{l}_{\mathrm{f}}$ or vectors $\vec{l}_{\mathrm{f}}$ and $\vec{l}_{\mathrm{a}}$ respectively. $\Phi_{j}$ is the angle of internal rotation for bond $j$. That is, $\Phi_{\mathrm{a}}$ and $\Phi_{\mathrm{f}}$ are the angle for bonds a and $f$ respectively. Therefore the distance $r_{i j}$ can be expressed by maxices $T_{j}$ and $\vec{l}_{j}$. In accordance with the RIS theory, ${ }^{1}$ the average value of $S^{2}$, which is expressed by $\left\langle S^{2}\right\rangle$, may be calculated by the statistical weight matrix $u_{j}$ depended on the conformation of the polymer. The conformation for some polymers, depend on the rotational angle $\Phi_{j}$, is described by three-state model, one for the trans $(t)$ and two for the gauche $\left(g^{+}, g^{-}\right)$. The internal rotation of the side bonds may be ignored. Then, two statistical weight matrices $u_{\mathrm{a}}$ and $u_{\mathrm{f}}$, depended on the states of $\Phi_{\mathrm{a}}$ and $\Phi_{\mathrm{f}}$, are enough to describe the statistical characteristics of the chain in Figure 1(a). Therefore, $\left\langle S^{2}\right\rangle$ is

$$
\left\langle S^{2}\right\rangle=M^{-2}\left(\left\langle S_{1}^{2}\right\rangle+\left\langle S_{2}^{2}\right\rangle+\left\langle S_{3}^{2}\right\rangle\right)
$$

where

$$
\begin{aligned}
\left\langle S_{1}^{2}\right\rangle= & \sum_{0 \leq i<j \leq 2 x} m_{i} m_{j}\left\langle r_{i j}^{2}\right\rangle \\
\left\langle S_{2}^{2}\right\rangle= & \left(\sum_{i=0}^{2 x} \sum_{j=2 x+1}^{4 x}\right. \\
& \left.+\sum_{i=2 x+1}^{3 x} \sum_{j=3 x+1}^{4 x}\right) m_{i} m_{j}\left\langle r_{i j}^{2}\right\rangle \\
\left\langle S_{3}^{2}\right\rangle= & \sum_{2 x+1 \leq i<j \leq 4 x} m_{i} m_{j}\left\langle r_{i j}^{2}\right\rangle
\end{aligned}
$$

$\left\langle S_{1}^{2}\right\rangle$ only relates to the skeletal atoms. Following Flory, ${ }^{1,2}$ it has been given as

$$
\left\langle S_{1}^{2}\right\rangle=2 Z^{-1} F^{\ddagger} G_{1}^{(2 x)} F=2 Z^{-1} F^{\ddagger}\left(G_{\mathrm{a}} G_{\mathrm{f}}\right)^{x} F
$$

where

$$
\begin{aligned}
& F^{\ddagger}=\left[\begin{array}{llll}
1 & 0 & \cdots & 0
\end{array}\right] \quad F=\left[\begin{array}{llllll}
0 & \cdots & 0 & 1 & 1 & 1
\end{array}\right]^{T} \\
& G_{j}=\left[\begin{array}{ccc}
u_{j} & m_{j-1} P g_{j} & m_{j-1} m_{j}\left(l_{j}^{2} / 2\right) u_{j} \\
0 & g_{j} & m_{j} g_{j} Q \\
0 & 0 & u_{j}
\end{array}\right]
\end{aligned}
$$

$F^{\ddagger}, F$, and $G_{j}$ are matrices with $1 \times 21,21 \times 1$, and $21 \times 21$ orders respectively. $j$ is taken $1,3,5 \cdots 2 x-1$ for the atom a and $0,2 \cdots 2 x$ for the atom $\mathrm{f}$. The partition function $Z$ is

$$
Z=J^{\ddagger} u_{1}^{(2 x)} J=J^{\ddagger}\left(u_{\mathrm{a}} u_{\mathrm{f}}\right)^{x} J
$$

where

$$
J^{\ddagger}=\left[\begin{array}{lll}
1 & 0 & 0
\end{array}\right] \quad J=\left[\begin{array}{lll}
1 & 1 & 1
\end{array}\right]^{T}
$$




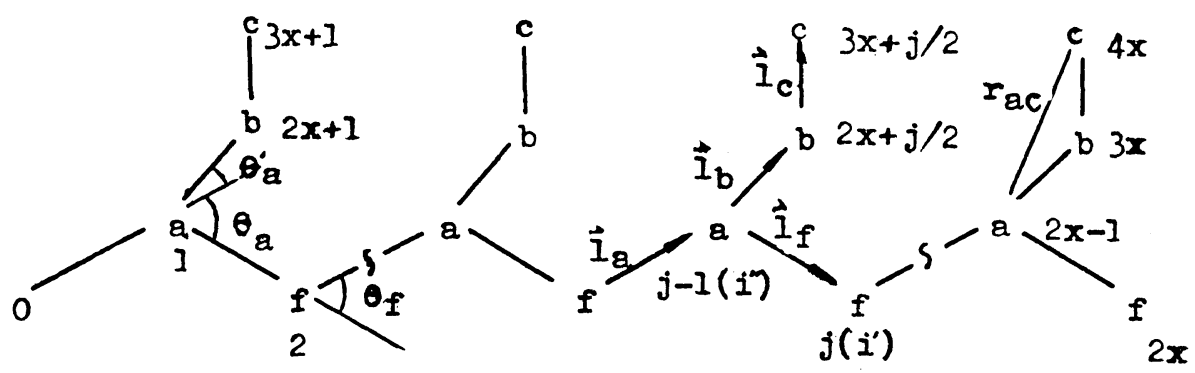

(a)

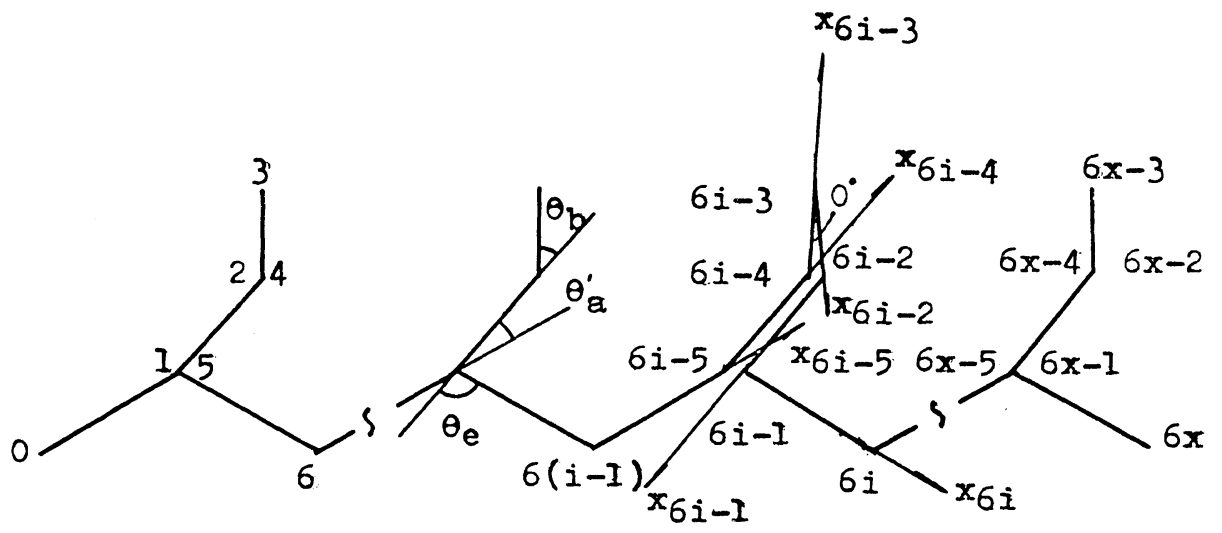

(b)

Figure 1. (a): Diagrammatic representation of the monosubstituted polymer with two side groups. (b): Diagrammatic representation of the consecutive Cartesian reference frames affixed to bonds.

$$
\begin{aligned}
g_{j} & =\left[\begin{array}{ccc}
u_{j} & \left(u_{j} \otimes \bar{l}_{j}^{T}\right)\|T\|_{j} & \left(l_{j}^{2} / 2\right) u_{j} \\
0 & \left(u_{j} \otimes E_{3}\right)\|T\|_{j} & u_{j} \otimes \bar{l}_{j} \\
0 & 0 & u_{j}
\end{array}\right] \\
& =g\left(u_{j}, l_{j}, T_{j}\right) \\
\|T\|_{j} & =\left[\begin{array}{ccc}
T\left(\Phi_{t}\right) & 0 & 0 \\
0 & T\left(\Phi_{g^{+}}\right) & 0 \\
0 & 0 & T\left(\Phi_{g^{-}}\right)
\end{array}\right] j
\end{aligned}
$$

$J^{\ddagger}, J, g_{j}$, and $\|T\|_{j}$ are matrices with $1 \times 3$, $3 \times 1,15 \times 15$, and $9 \times 9$ orders.

The expressions of $\left\langle S_{2}^{2}\right\rangle$ and $\left\langle S_{3}^{2}\right\rangle$ must take account of the structure of the side groups in every repeat units. The mark from 0 to $4 x$ in Figure 1(a) are renumered serially from 0 to $6 x$ in Figure 1(b). Similarly, new Cartesian reference frames $\left\{x_{j}, y_{j}, z_{j}\right\}$ affixed to the skeletal bonds and side group bonds are founded as shown Figure 1(b), where $j$ is taken with $6 i-5,6 i-4,6 i-3,6 i-2,6 i-1$, and $6 i$ (or expressed as a, b, c, d, e, and f) respectively in the $i$-th repeat unit, and $i$ is taken from 1 to $x$. Let the axis $x_{6 i-5}, x_{6 i-4}, x_{6 i-3}$, and $x_{6 i}$ of the consecutive coordinate systems affixed to the bonds be in the direction of the bond vectors $\vec{l}_{\mathrm{a}}, \vec{l}_{\mathrm{b}}, \vec{l}_{\mathrm{c}}$, and $\vec{l}_{\mathrm{f}}$, respectively. The axis $x_{6 i-2}$ and $x_{6 i-1}$ affixed to the bonds $\mathrm{b}$ and $\mathrm{c}$ in opposite direction of the bond vectors $\vec{l}_{\mathrm{b}}$ and $\vec{l}_{\mathrm{c}}$, respectively. The axis $y_{j}$ and $z_{j}$ are defined by the similar way to the RIS theory. Then, $\theta_{a}^{\prime}$ is the angle between axis $x_{6 i-5}$ and $x_{6 \mathrm{i}-4} ; \theta_{\mathrm{b}}$ is that between axis $x_{6 i-4}$ and $x_{6 i-3}$ or $x_{6 i-2}$ and $x_{6 i-1}$; and $\theta_{\mathrm{e}}$ is that between $x_{6 i-1}$ and $x_{6 i} . \Phi_{j}$ may be separated into two parts, $\Phi_{j}$ and $\Phi_{j}^{\prime}$. 
The former describes the states of internal rotation of bond $j$. The latter, depended on the geometric structure of the polymer, denotes the dihedral angle between plan $x_{j} y_{j}$ and plan $x_{j+1} y_{j+1}$ when $\Phi_{j}$ is in trans. Therefore, in each repeat unit, the axis transformation matrix $T_{j}$ relating to consecutive coordinate systems is given by eq 4 as

$$
\begin{aligned}
& T_{6 i-5}=T_{\mathrm{a}}=T\left(\theta_{\mathrm{a}}^{\prime}, \Phi_{\mathrm{a}}+\Phi_{\mathrm{a}}^{\prime}\right) \\
& T_{6 i-4}=T_{\mathrm{b}}=T\left(\theta_{\mathrm{b}}, \Phi_{\mathrm{b}}^{\prime}\right) \\
& T_{6 i-3}=T_{\mathrm{c}}=T\left(180^{\circ}, 0^{\circ}\right) \\
& T_{6 i-2}=T_{\mathrm{d}}=T\left(\theta_{\mathrm{b}}, 180^{\circ}\right) \\
& T_{6 i-1}=T_{\mathrm{e}}=T\left(\theta_{\mathrm{e}}, \Phi_{\mathrm{e}}^{\prime}\right) \\
& T_{6 i}=T_{\mathrm{f}}=T\left(\theta_{\mathrm{f}}, \Phi_{\mathrm{f}}+\Phi_{\mathrm{f}}^{\prime}\right) \\
& \quad i=1,2,3 \cdots x
\end{aligned}
$$

where $\Phi_{\mathrm{a}}^{\prime}$ and $\Phi_{\mathrm{f}}^{\prime}$ can be calculated by

$\Phi_{\mathrm{b}}^{\prime}$ and $\Phi_{\mathrm{e}}^{\prime}$ are correlative to the dihedral angle between the plan of side groups and the plan of the skeletal bonds in all-trans, and $\Phi_{\mathrm{b}}^{\prime}$ and $\Phi_{\mathrm{e}}^{\prime}$ are connected by

$\cos \theta_{\mathrm{a}}^{\prime} \cos \theta_{\mathrm{e}} \cos ^{2} \theta_{\mathrm{b}}+\sin \theta_{\mathrm{a}}^{\prime} \sin \theta_{\mathrm{e}} \sin ^{2} \theta_{\mathrm{b}}+\sin \theta_{\mathrm{a}}^{\prime} \sin \theta_{\mathrm{e}}\left(\cos \Phi_{\mathrm{b}}^{\prime} \cos \Phi_{\mathrm{e}}^{\prime}+\sin \Phi_{\mathrm{b}}^{\prime} \sin \Phi_{\mathrm{e}}^{\prime}\right)=-\cos \theta_{\mathrm{a}}$

Moreover, $\Phi_{j}^{\prime}$ may be taken with the same angles in all repeat units for isotactic polymers, and with the different angles in the alternating units for syndiotactic polymers, even with the angles of probability in the different units for atactic polymers by using the computer simulation. Because of ignoring internal rotation of bonds $\mathrm{b}$ and $\mathrm{c}$, i.e.,

$$
\Phi_{j}=0 \quad(j=6 i-4,6 i-3,6 i-2,6 i-1 \text { or } \mathrm{b}, \mathrm{c}, \mathrm{d}, \mathrm{e})
$$

then, the statistical weight matrix $u_{j}$ is

$$
\begin{array}{ll}
u_{6 i-5}=u_{\mathrm{a}} & (i=2,3, \cdots x) \\
u_{j}=E_{3} & (j=6 i-4,6 i-3,6 i-2,6 i-1,1,6 x) \\
u_{6 i}=u_{\mathrm{f}} & (i=1,2, \cdots x-1)
\end{array}
$$

with $E_{3}$ being the identity matrix of orders 3 . Using the method of matrix algebra, $\left\langle S_{2}^{2}\right\rangle$ can be derivated by

$$
\begin{aligned}
\left\langle S_{2}^{2}\right\rangle= & \left(\sum_{i=0}^{2 x} \sum_{j=2 x+1}^{4 x}+\sum_{i=2 x+1}^{3 x} \sum_{j=3 x+1}^{4 x}\right) m_{i} m_{j}\left\langle r_{i j}^{2}\right\rangle=\left(m_{\mathrm{f}} m_{\mathrm{b}} \sum_{i^{\prime}=0}^{x} \sum_{j=2 x+1}^{3 x}+m_{\mathrm{f}} m_{\mathrm{c}} \sum_{i^{\prime}=0}^{x} \sum_{j=3 x+1}^{4 x}\right)\left\langle r_{i j}^{2}\right\rangle \\
& +\left(m_{\mathrm{a}} m_{\mathrm{b}} \sum_{i^{\prime \prime}=1}^{x} \sum_{j=2 x+1}^{3 x}+m_{\mathrm{a}} m_{\mathrm{c}} \sum_{i^{\prime \prime}=1}^{x} \sum_{j=3 x+1}^{4 x}\right)\left\langle r_{i j}^{2}\right\rangle+m_{\mathrm{b}} m_{\mathrm{c}} \sum_{i=2 x+1}^{3 x} \sum_{j=3 x+1}^{4 x}\left\langle r_{i j}^{2}\right\rangle
\end{aligned}
$$

where

$$
\left.\begin{array}{ll}
i^{\prime}=j / 2 & (j=0,2,4 \cdots 2 x) \\
i^{\prime \prime}=(j+1) / 2 & (j=1,3,5 \cdots 2 x-1)
\end{array}\right\}
$$

Following Figure 1(b), $\left\langle S_{2}^{2}\right\rangle$ is also expressed as 


$$
\begin{aligned}
\left\langle S_{2}^{2}\right\rangle= & m_{\mathrm{f}} m_{\mathrm{b}} \sum_{h=0}^{x} \sum_{k=1}^{x}\left\langle r_{6 h, 6 k-4}^{2}\right\rangle+m_{\mathrm{f}} m_{\mathrm{c}} \sum_{h=0}^{x} \sum_{k=1}^{x}\left\langle r_{6 h, 6 k-3}^{2}\right\rangle \\
& +m_{\mathrm{a}} m_{\mathrm{b}} \sum_{h=1}^{x} \sum_{k=1}^{x}\left\langle r_{6 h-5,6 k-4}^{2}\right\rangle+m_{\mathrm{a}} m_{\mathrm{c}} \sum_{h=1}^{x} \sum_{k=1}^{x}\left\langle r_{6 h-5,6 k-3}^{2}\right\rangle \\
& +m_{\mathrm{b}} m_{\mathrm{c}} \sum_{h=1}^{x} \sum_{k=1}^{x}\left\langle r_{6 h-4,6 k-3}^{2}\right\rangle \\
= & 4 m_{\mathrm{f}} m_{\mathrm{b}} Z^{-1} J^{\ddagger} \sum_{0 \leq h<k \leq x} u_{1}^{(6 h)} P g_{6 h+1}^{(6 k-6 h-4)} Q u_{6 k-3}^{(6 x-6 k+4)} J \\
& +4 m_{\mathrm{f}} m_{\mathrm{c}} Z^{-1} J^{\ddagger} \sum_{0 \leq h<k \leq x} u_{1}^{(6 h)} P g_{6 h+1}^{(6 k-6 h-3)} Q u_{6 k-2}^{(6 x-6 k+3)} J \\
& +m_{\mathrm{a}} m_{\mathrm{b}}\left[\sum_{h=1}^{x} r_{6 h-5,6 k-4}^{2}+4 Z^{-1} J^{\neq} \sum_{1 \leq h<k \leq x} u_{1}^{(6 h-5)} P g_{6 h-4}^{(6 k-6 h+1)} Q u_{6 k-3}^{(6 x-6 k+4)} J\right] \\
& +m_{\mathrm{a}} m_{\mathrm{c}}\left[\sum_{h=1}^{x} r_{6 h-5,6 k-3}^{2}+4 Z^{-1} J^{\neq} \sum_{1 \leq h<k \leq x} u_{1}^{(6 h-5)} P g_{6 h-4}^{(6 k-6 h+2)} Q u_{6 k-2}^{(6 x-6 k+3)} J\right] \\
& +m_{\mathrm{b}} m_{\mathrm{c}}\left[\sum_{h=1}^{x} r_{6 h-4,6 k-3}^{2}+4 Z^{-1} J^{\ddagger} \sum_{1 \leq h<k \leq x} u_{1}^{(6 h-4)} P g_{6 h-3}^{(6 k-6 h+1)} Q u_{6 k-2}^{(6 x-6 k+3)} J\right]
\end{aligned}
$$

where

$$
P=\left[\begin{array}{ll}
E_{3} & 0
\end{array}\right], \quad Q=\left[\begin{array}{ll}
0 & E_{3}
\end{array}\right]^{T}
$$

$P$ and $Q$ are matrices of $3 \times 15$ and $15 \times 3$ orders, respectively. The first terms in eq 26 can be expressed by $4 m_{\mathrm{f}} m_{\mathrm{b}} Z^{-1} F^{\ddagger} S(1)_{1}^{(x)} F$, where

$$
S(1)_{i}=\left[\begin{array}{ccc}
u_{6 i-5}^{(6)} & P g_{6 i-5}^{(6)} & P g_{6 i-5}^{(2)} Q u_{6 i-3}^{(4)} \\
0 & g_{6 i-5}^{(6)} & g_{6 i-5}^{(2)} Q u_{6 i-3}^{(4)} \\
0 & 0 & u_{6 i-5}^{(6)}
\end{array}\right]
$$

and $g_{j}$ from eq 14 may be expressed by

$$
\begin{aligned}
& g_{6 i-5}=g_{\mathrm{a}}=g\left(u_{\mathrm{a}}, l_{\mathrm{a}}, T_{\mathrm{a}}\right) \\
& g_{6 i-4}=g_{\mathrm{b}}=g\left(E_{3}, l_{\mathrm{b}}, T_{\mathrm{b}}\right) \\
& g_{6 i-3}=g_{\mathrm{c}}=g\left(E_{3}, l_{\mathrm{c}}, T_{\mathrm{c}}\right) \\
& g_{6 i-2}=g_{\mathrm{d}}=g\left(E_{3}, l_{\mathrm{c}}, T_{\mathrm{d}}\right) \\
& g_{6 i-1}=g_{\mathrm{e}}=g\left(E_{3}, l_{\mathrm{b}}, T_{\mathrm{e}}\right) \\
& g_{6 i}=g_{\mathrm{f}}=g\left(u_{f}, l_{\mathrm{f}}, T_{\mathrm{f}}\right)
\end{aligned}
$$

moreover, $S(1)_{i}$ may be identified with

$$
S(1)=\left[\begin{array}{ccc}
u_{\mathrm{a}} u_{\mathrm{f}} & P g_{\mathrm{a}} g_{\mathrm{b}} g_{\mathrm{c}} g_{\mathrm{d}} g_{\mathrm{e}} g_{\mathrm{f}} & P g_{\mathrm{a}} g_{\mathrm{b}} Q u_{\mathrm{f}} \\
0 & g_{\mathrm{a}} g_{\mathrm{b}} g_{\mathrm{c}} g_{\mathrm{d}} g_{\mathrm{e}} g_{\mathrm{f}} & g_{\mathrm{a}} g_{\mathrm{b}} Q u_{\mathrm{f}} \\
0 & 0 & u_{\mathrm{a}} u_{\mathrm{f}}
\end{array}\right]
$$


Similar to the other terms in eq 26 , then, $\left\langle S_{2}^{2}\right\rangle$ can be given

$$
\begin{aligned}
\left\langle S_{2}^{2}\right\rangle= & 4 Z^{-1}\left[m_{\mathrm{f}} m_{\mathrm{b}} F^{\ddagger} S(1)^{x} F+m_{\mathrm{f}} m_{\mathrm{c}} F^{\ddagger} S(2)^{x} F\right. \\
& \left.+m_{\mathrm{a}} m_{\mathrm{b}} F^{\ddagger} S(3)^{x} F+m_{\mathrm{a}} m_{\mathrm{c}} F^{\ddagger} S(4)^{x} F+m_{\mathrm{b}} m_{\mathrm{c}} F^{\ddagger} S(5)^{x} F\right]
\end{aligned}
$$

where

$$
\begin{aligned}
& S(2)=\left[\begin{array}{ccc}
u_{\mathrm{a}} u_{\mathrm{f}} & P g_{\mathrm{a}} g_{\mathrm{b}} g_{\mathrm{c}} g_{\mathrm{d}} g_{\mathrm{e}} g_{\mathrm{f}} & P g_{\mathrm{a}} g_{\mathrm{b}} g_{\mathrm{c}} Q u_{\mathrm{f}} \\
0 & g_{\mathrm{a}} g_{\mathrm{b}} g_{\mathrm{d}} g_{\mathrm{c}} g_{\mathrm{e}} g_{\mathrm{f}} & g_{\mathrm{a}} g_{\mathrm{b}} g_{\mathrm{c}} Q u_{\mathrm{f}} \\
0 & 0 & u_{\mathrm{a}} u_{\mathrm{f}}
\end{array}\right] \\
& S(3)=\left[\begin{array}{ccc}
u_{\mathrm{a}} u_{\mathrm{f}} & u_{\mathrm{a}} P g_{\mathrm{b}} g_{\mathrm{c}} g_{\mathrm{d}} g_{\mathrm{e}} g_{\mathrm{f}} & \left(l_{\mathrm{b}}^{2} / 4\right) u_{\mathrm{a}} u_{\mathrm{f}} \\
0 & g_{\mathrm{a}} g_{\mathrm{b}} g_{\mathrm{c}} g_{\mathrm{d}} g_{\mathrm{e}} g_{\mathrm{f}} & g_{\mathrm{a}} g_{\mathrm{b}} Q u_{\mathrm{f}} \\
0 & 0 & u_{\mathrm{a}} u_{\mathrm{f}}
\end{array}\right] \\
& S(4)=\left[\begin{array}{ccc}
u_{\mathrm{a}} u_{\mathrm{f}} & u_{\mathrm{a}} P g_{\mathrm{b}} g_{\mathrm{c}} g_{\mathrm{d}} g_{\mathrm{e}} g_{\mathrm{f}}\left(r_{\mathrm{ac}}^{2} / 4\right) u_{\mathrm{a}} u_{\mathrm{f}} \\
0 & g_{\mathrm{a}} g_{\mathrm{b}} g_{\mathrm{c}} g_{\mathrm{d}} g_{\mathrm{e}} g_{\mathrm{f}} & g_{\mathrm{a}} g_{\mathrm{b}} g_{\mathrm{c}} Q u_{\mathrm{f}} \\
0 & 0 & u_{\mathrm{a}} u_{\mathrm{f}}
\end{array}\right] \\
& S(5)=\left[\begin{array}{ccc}
u_{\mathrm{a}} u_{\mathrm{f}} & u_{\mathrm{a}} P g_{\mathrm{c}} g_{\mathrm{d}} g_{\mathrm{e}} g_{\mathrm{f}} & \left(l_{\mathrm{c}}^{2} / 4\right) u_{\mathrm{a}} u_{\mathrm{f}} \\
0 & g_{\mathrm{a}} g_{\mathrm{b}} g_{\mathrm{c}} g_{\mathrm{d}} g_{\mathrm{e}} g_{\mathrm{f}} & g_{\mathrm{a}} g_{\mathrm{b}} g_{\mathrm{c}} Q u_{\mathrm{f}} \\
0 & 0 & u_{\mathrm{a}} u_{\mathrm{f}}
\end{array}\right] \\
& r_{\mathrm{ac}}^{2}=l_{\mathrm{b}}^{2}+l_{\mathrm{c}}^{2}+2 l_{\mathrm{b}} l_{\mathrm{c}} \cos \theta_{\mathrm{b}}
\end{aligned}
$$

they are matrices of orders $21 \times 21$. Similarly, $\left\langle S_{3}^{2}\right\rangle$ can be derivated by

$$
\begin{aligned}
\left\langle S_{3}^{2}\right\rangle= & \left(\sum_{2 x+1 \leq i<j \leq 3 x}+\sum_{3 x+1 \leq i<j \leq 4 x}\right) m_{i} m_{j}\left\langle r_{i j}^{2}\right\rangle \\
= & m_{\mathrm{b}}^{2} \sum_{1 \leq h<k \leq x}\left\langle r_{6 h-4,6 k-4}^{2}\right\rangle+m_{\mathrm{c}}^{2} \sum_{1 \leq h<k \leq x}\left\langle r_{6 h-3,6 k-3}^{2}\right\rangle \\
= & 2 Z^{-1}\left[m_{\mathrm{b}}^{2} J^{\ddagger} \sum_{1 \leq h<k \leq x} u_{1}^{(6 h-4)} P g_{6 h-3}^{(6 k-6 h)} Q u_{6 k-3}^{(6 x-6 k+4)} J\right. \\
& \left.+m_{\mathrm{c}}^{2} J^{\ddagger} \sum_{1 \leq h<k \leq x} u_{1}^{(6 h-3)} P g_{6 h-2}^{(6 k-6 h)} Q u_{6 k-2}^{(6 x-6 k+3)} J\right] \\
= & 2 Z^{-1}\left[m_{\mathrm{b}}^{2} F^{\ddagger} S(6)^{x} F+m_{\mathrm{c}}^{2} F^{\ddagger} S(7)^{x} F\right]
\end{aligned}
$$

where

$$
S(6)=\left[\begin{array}{ccc}
u_{\mathrm{a}} u_{\mathrm{f}} & u_{\mathrm{a}} P g_{\mathrm{c}} g_{\mathrm{d}} g_{\mathrm{e}} g_{\mathrm{f}} & 0 \\
0 & g_{\mathrm{a}} g_{\mathrm{b}} g_{\mathrm{c}} g_{\mathrm{d}} g_{\mathrm{e}} g_{\mathrm{f}} & g_{\mathrm{a}} g_{\mathrm{b}} Q u_{\mathrm{f}} \\
0 & 0 & u_{\mathrm{a}} u_{\mathrm{f}}
\end{array}\right] \quad S(7)=\left[\begin{array}{ccc}
u_{\mathrm{a}} u_{\mathrm{f}} & u_{\mathrm{a}} P g_{\mathrm{d}} g_{\mathrm{e}} g_{\mathrm{f}} & 0 \\
0 & g_{\mathrm{a}} g_{\mathrm{b}} g_{\mathrm{c}} g_{\mathrm{d}} g_{\mathrm{e}} g_{\mathrm{f}} & g_{\mathrm{a}} g_{\mathrm{b}} g_{\mathrm{c}} Q u_{\mathrm{f}} \\
0 & 0 & u_{\mathrm{a}} u_{\mathrm{f}}
\end{array}\right]
$$

(38) Therefore, the unperturbed mean-square ra- 
dius of gyration of the polymer chains, such as isotactic and syndiotactic 1,2-PBD, can be calculated by eq 5-39 or by the following equation

$$
\begin{aligned}
\left\langle S^{2}\right\rangle= & 2 Z^{-1} M^{-2} F^{\ddagger}\left(G_{\mathrm{a}} G_{\mathrm{f}}\right)^{x} F \\
& +2 Z^{-1} M^{-2} \sum_{q=1}^{7} m(q) F^{\ddagger} S(q)^{x} F
\end{aligned}
$$

where $m(1)-m(7)$ replace $2 m_{\mathrm{f}} m_{\mathrm{b}}, 2 m_{\mathrm{f}} m_{\mathrm{c}}$, $2 m_{\mathrm{a}} m_{\mathrm{c}}, 2 m_{\mathrm{a}} m_{\mathrm{c}}, 2 m_{\mathrm{b}} m_{\mathrm{c}}, m_{\mathrm{b}}^{2}$, and $m_{\mathrm{c}}^{2}$, respectively. The first term in eq 40 , only related to the skeletal atoms of the polymer, is derivated without considering the side groups. The second term may be regarded as the revision with considering the geometric structure of side groups. The expression 40 is easy to be executed numerical calculation.

If the geometric structure of the second side groups for the polymer chain is ignored, eq 40 can be reduced to that of the polymer chains with single side group as follows: The first, the mass $m_{\mathrm{b}}$ is assumed to be zero, eq 40 may be simplified to

$$
\begin{aligned}
\left\langle S^{2}\right\rangle= & 2 Z^{-1} M^{-2} F^{\ddagger}\left[\left(G_{\mathrm{a}} G_{\mathrm{f}}\right)^{x}+2 m_{\mathrm{f}} m_{\mathrm{c}} S(2)^{x}\right. \\
& \left.+2 m_{\mathrm{a}} m_{\mathrm{c}} S(4)^{x}+m_{\mathrm{c}}^{2} S(7)^{x}\right] F
\end{aligned}
$$

The second, the structure of side groups is changed into one side group instead of two side groups. Let vector $\vec{l}_{\mathrm{c}}$ be taken in the direction of vector $\vec{l}_{\mathrm{b}}$, i.e., $\theta_{\mathrm{b}}$ and $\Phi_{\mathrm{b}}$ are regarded as $0^{\circ}$ and $180^{\circ}$ respectively, or the axis transformation matrices $T_{\mathrm{b}}$ and $T_{\mathrm{d}}$ become the identity matrix. Then, $\Phi_{\mathrm{e}}^{\prime}$ given by eq 19 as

$$
\cos \Phi_{\mathrm{e}}^{\prime}=\left(\cos \theta_{\mathrm{a}}+\cos \theta_{\mathrm{e}} \cos \theta_{\mathrm{a}}^{\prime}\right) / \sin \theta_{\mathrm{e}} \sin \theta_{\mathrm{a}}^{\prime}
$$

The third, the bond length $l_{\mathrm{b}}$ is regarded as zero. Substituting eq 16-18 and 42 into eq 29, $g_{j}$ can be expressed by

$$
\begin{aligned}
& g_{\mathrm{a}}=g\left(u_{\mathrm{a}}, l_{\mathrm{a}}, T_{\mathrm{a}}\right) \equiv g_{\alpha} \\
& g_{\mathrm{b}}=g\left(E_{3}, 0, E_{3}\right)=E_{15} \\
& g_{\mathrm{c}}=g\left(E_{3}, l_{\mathrm{c}}, T_{\mathrm{c}}\right) \equiv g_{\beta} \\
& g_{\mathrm{d}}=g\left(E_{3}, l_{\mathrm{c}}, E_{3}\right) \\
& g_{\mathrm{e}}=g\left(E_{3}, 0, T_{\mathrm{e}}\right) \\
& g_{\mathrm{f}}=g\left(u_{\mathrm{f}}, l_{\mathrm{f}}, T_{\mathrm{f}}\right)=g_{\delta}
\end{aligned}
$$

where $E_{15}$ is the identity matrix of orders 15 . Moreover, we have

$$
g_{\mathrm{d}} g_{\mathrm{e}}=g\left(E_{3}, l_{\mathrm{c}}, T_{\mathrm{e}}\right) \equiv g_{\gamma}
$$

Then, $S(2), S(4)$, and $S(7)$ can be simplified to

$$
\begin{gathered}
S(2)=\left[\begin{array}{ccc}
u_{\mathrm{a}} u_{\mathrm{f}} & P g_{\alpha} g_{\beta} g_{\gamma} g_{\delta} & P g_{\alpha} g_{\beta} Q u_{\mathrm{f}} \\
0 & g_{\alpha} g_{\beta} g_{\gamma} g_{\delta} & g_{\alpha} g_{\beta} Q u_{\mathrm{f}} \\
0 & 0 & u_{\mathrm{a}} u_{\mathrm{f}}
\end{array}\right] \\
S(4)=\left[\begin{array}{ccc}
u_{\mathrm{a}} u_{\mathrm{f}} & u_{\mathrm{a}} P g_{\beta} g_{\gamma} g_{\delta} & \left(l_{\mathrm{c}}^{2} / 4\right) u_{\mathrm{a}} u_{\mathrm{f}} \\
0 & g_{\alpha} g_{\beta} g_{\gamma} g_{\delta} & g_{\alpha} g_{\beta} Q u_{\mathrm{f}} \\
0 & 0 & u_{\mathrm{a}} u_{\mathrm{f}}
\end{array}\right]
\end{gathered}
$$

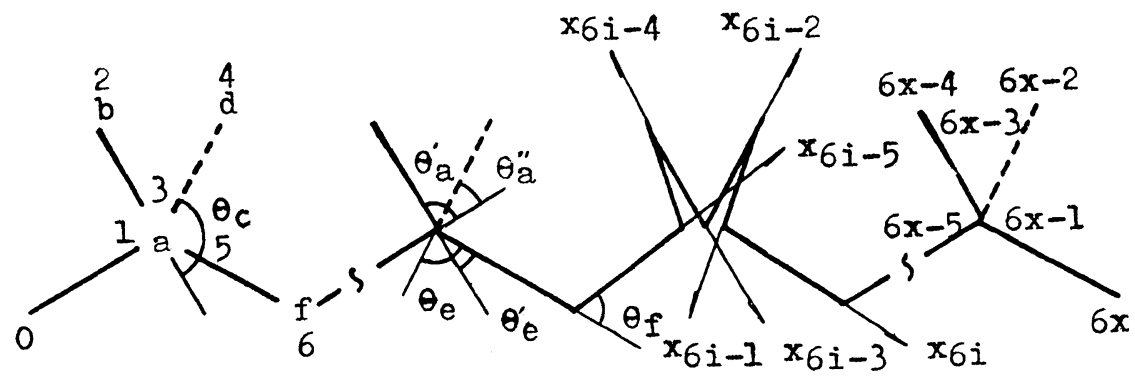

Figure 2. Diagrammatic representation of the consecutive Cartesian reference frames affixed to the bonds for the polymer, such as PDMS. 


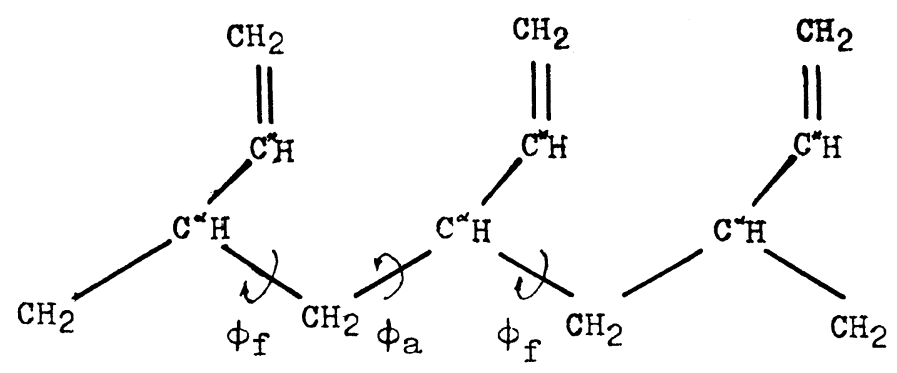

Figure 3. Schematic representation of the isotactic 1,2-polybutadiene chain in the all-trans.

$$
S(7)=\left[\begin{array}{ccc}
u_{\mathrm{a}} u_{\mathrm{f}} & u_{\mathrm{a}} P g_{\gamma} g_{\delta} & 0 \\
0 & g_{\alpha} g_{\beta} g_{\gamma} g_{\delta} & g_{\alpha} g_{\beta} Q u_{\mathrm{f}} \\
0 & 0 & u_{\mathrm{a}} u_{\mathrm{f}}
\end{array}\right]
$$

Therefore, eq 41 can be investigated the meansquare radius of gyration of the polymers with single side group, such as isotactic and syndiotactic PP.

The monosubstituted polymer chain shown in Figure 3 is also investigated by eq 40 . The mass $m_{j}$, the bond length $l_{j}$ and the angle $\theta_{j}$ are defined as above or shown in Figure 2. The axis transformation matrix $T_{j}$ is given by

$$
\begin{aligned}
& T_{6 i-5}=T_{\mathrm{a}}=T\left(\theta_{\mathrm{a}}^{\prime}, \Phi_{\mathrm{a}}+\Phi_{\mathrm{a}}^{\prime}\right) \\
& T_{6 i-4}=T_{\mathrm{b}}=T\left(180^{\circ}, 0^{\circ}\right) \\
& T_{6 i-3}=T_{\mathrm{c}}=T\left(\theta_{\mathrm{c}}, \Phi_{\mathrm{c}}^{\prime}\right) \\
& T_{6 i-2}=T_{\mathrm{d}}=T\left(180^{\circ}, 0^{\circ}\right) \\
& T_{6 i-1}=T_{\mathrm{e}}=T\left(\theta_{\mathrm{e}}, \Phi_{\mathrm{e}}^{\prime}\right) \\
& T_{6 i}=T_{\mathrm{f}}=T\left(\theta_{\mathrm{f}}, \Phi_{\mathrm{f}}+\Phi_{\mathrm{f}}^{\prime}\right)
\end{aligned}
$$

where $\Phi_{\mathrm{a}}^{\prime}, \Phi_{\mathrm{c}}^{\prime}, \Phi_{\mathrm{e}}^{\prime}$, and $\Phi_{\mathrm{f}}^{\prime}$ can be calculated by $\cos \Phi_{\mathrm{a}}^{\prime}=-\left(\cos \theta_{\mathrm{c}}+\cos \theta_{\mathrm{a}}^{\prime} \cos \theta_{\mathrm{a}}^{\prime \prime}\right) / \sin \theta_{\mathrm{a}}^{\prime} \sin _{\mathrm{a}}^{\prime \prime}$

$\cos \Phi_{\mathrm{c}}^{\prime}=\left(\cos \theta_{\mathrm{a}}^{\prime \prime}+\cos \theta_{\mathrm{a}}^{\prime} \cos \theta_{\mathrm{a}}\right) / \sin \theta_{\mathrm{a}}^{\prime} \sin \theta_{\mathrm{c}}$

$\cos \Phi_{\mathrm{e}}^{\prime}=\left(\cos \theta_{\mathrm{e}}^{\prime}+\cos \theta_{\mathrm{c}} \cos \theta_{\mathrm{e}}\right) / \sin \theta_{\mathrm{c}} \sin \theta_{\mathrm{e}}$

$\cos \Phi_{\mathrm{f}}^{\prime}=-\left(\cos \theta_{\mathrm{f}}^{\prime \prime}+\cos \theta_{\mathrm{f}} \cos \theta_{\mathrm{e}}\right) / \sin \theta_{\mathrm{e}} \sin \theta_{\mathrm{f}}$ where $\theta_{\mathrm{a}}^{\prime \prime}$ is angle between axis $x_{6 i-5}$ and $x_{6 i-2}$, $\theta_{\mathrm{e}}^{\prime}$ is angle between $x_{6 i-3}$ and $x_{6 i}$. Then, $g_{j}$ may be expressed by

$$
\left.\begin{array}{l}
g_{6 i-5}=g_{\mathrm{a}}=g\left(u_{\mathrm{a}}, l_{\mathrm{a}}, T_{\mathrm{a}}\right) \\
g_{6 i-4}=g_{\mathrm{b}}=g\left(E_{3}, l_{\mathrm{b}}, T_{\mathrm{b}}\right) \\
g_{6 i-3}=g_{\mathrm{c}}=g\left(E_{3}, l_{\mathrm{b}}, T_{\mathrm{c}}\right) \\
g_{6 i-2}=g_{\mathrm{d}}=g\left(E_{3}, l_{\mathrm{d}}, T_{\mathrm{d}}\right) \\
g_{6 i-1}=g_{\mathrm{e}}=g\left(E_{3}, l_{\mathrm{d}}, T_{\mathrm{e}}\right) \\
g_{6 i}=g_{\mathrm{f}}=g\left(u_{\mathrm{f}}, l_{\mathrm{f}}, T_{\mathrm{f}}\right)
\end{array}\right\}
$$

where the statistical weight matrix $u_{j}$ is also given by eq $21-23$. The expression of $S(1)$, $S(3)$, and $S(6)$ are similar expressions to eq 30 , 33 , and 38 , respectively. Others of $S(q)$ are given

$$
S(2)=\left[\begin{array}{ccc}
u_{\mathrm{a}} u_{\mathrm{f}} & P g_{\mathrm{a}} g_{\mathrm{b}} g_{\mathrm{c}} g_{\mathrm{d}} g_{\mathrm{e}} g_{\mathrm{f}} & P g_{\mathrm{a}} g_{\mathrm{b}} g_{\mathrm{c}} g_{\mathrm{d}} Q u_{\mathrm{f}} \\
0 & g_{\mathrm{a}} g_{\mathrm{b}} g_{\mathrm{c}} g_{\mathrm{d}} g_{\mathrm{e}} g_{\mathrm{f}} & g_{\mathrm{a}} g_{\mathrm{b}} g_{\mathrm{c}} g_{\mathrm{d}} Q u_{\mathrm{f}} \\
0 & 0 & u_{\mathrm{a}} u_{\mathrm{f}}
\end{array}\right]
$$

$S(4)=\left[\begin{array}{ccc}u_{\mathrm{a}} u_{\mathrm{f}} & u_{\mathrm{a}} P g_{\mathrm{b}} g_{\mathrm{c}} g_{\mathrm{d}} g_{\mathrm{e}} g_{\mathrm{f}} & \left(l_{\mathrm{d}}^{2} / 4\right) u_{\mathrm{a}} u_{\mathrm{f}} \\ 0 & g_{\mathrm{a}} g_{\mathrm{b}} g_{\mathrm{c}} g_{\mathrm{d}} g_{\mathrm{e}} g_{\mathrm{f}} & g_{\mathrm{a}} g_{\mathrm{b}} g_{\mathrm{c}} g_{\mathrm{d}} Q u_{\mathrm{f}} \\ 0 & 0 & u_{\mathrm{a}} u_{\mathrm{f}}\end{array}\right]$

$S(5)=\left[\begin{array}{ccc}u_{\mathrm{a}} u_{\mathrm{f}} & u_{\mathrm{a}} P g_{\mathrm{c}} g_{\mathrm{d}} g_{\mathrm{e}} g_{\mathrm{f}} & \left(r_{\mathrm{bd}}^{2} / 4\right) u_{\mathrm{a}} u_{\mathrm{f}} \\ 0 & g_{\mathrm{a}} g_{\mathrm{b}} g_{\mathrm{c}} g_{\mathrm{d}} g_{\mathrm{e}} g_{\mathrm{f}} & g_{\mathrm{a}} g_{\mathrm{b}} g_{\mathrm{c}} g_{\mathrm{d}} Q u_{\mathrm{f}} \\ 0 & 0 & u_{\mathrm{a}} u_{\mathrm{f}}\end{array}\right]$ 
$S(7)=\left[\begin{array}{ccc}u_{\mathrm{a}} u_{\mathrm{f}} & u_{\mathrm{a}} P g_{\mathrm{e}} g_{\mathrm{f}} & 0 \\ 0 & g_{\mathrm{a}} g_{\mathrm{b}} g_{\mathrm{c}} g_{\mathrm{d}} g_{\mathrm{e}} g_{\mathrm{f}} & g_{\mathrm{a}} g_{\mathrm{b}} g_{\mathrm{c}} g_{\mathrm{d}} Q u_{\mathrm{f}} \\ 0 & 0 & u_{\mathrm{a}} u_{\mathrm{f}}\end{array}\right]$

where

$$
r_{\mathrm{bd}}^{2}=l_{\mathrm{d}}^{2}+l_{\mathrm{b}}^{2}+2 l_{\mathrm{b}} l_{\mathrm{d}} \cos \theta_{\mathrm{c}}
$$

$m(1)-m(7)$ in eq 40 replaces $2 m_{\mathrm{f}} m_{\mathrm{b}}, 2 m_{\mathrm{f}} m_{\mathrm{d}}$, $2 m_{\mathrm{a}} m_{\mathrm{b}}, 2 m_{\mathrm{a}} m_{\mathrm{d}}, 2 m_{\mathrm{b}} m_{\mathrm{d}}, m_{\mathrm{b}}^{2}$, and $m_{\mathrm{d}}^{2}$, respectively. Therefore the mean-square radius of gyration of the polymer with considering two side groups, such as PDMS, may be investigated by eq 40 , too.

If the structures of two side groups are ignored, or the masses of side groups $b$ and $c$ in Figure 1 (or $b$ and $d$ in Figure 2) are assumed to be zero, the second term of eq 40 becomes zero. Then, the expression is in agreement with that by Flory. ${ }^{1,2}$

\section{CALCULATION OF $\left\langle S^{2}\right\rangle$ FOR ISOTATIC 1,2-POLYBUTADIERE}

The unperturbed mean-square radius of gyration for one polymer depends on the conformational characteristics, the geomatric structure and the distribution of atom masses in a monomer. Such as isotactic 1,2-PBD shown in Figure 3, the conformation can be described by three-state model, the trans $\left(0^{\circ}\right)$ and the gauche $\left(120^{\circ}\right.$ and $\left.-120^{\circ}\right)$. The statistical weight matrices $u_{\mathrm{a}}$ and $u_{\mathrm{f}} \operatorname{are}^{11-15}$

$$
\begin{aligned}
& u_{\mathrm{a}}=\left[\begin{array}{ccc}
\eta \omega^{\prime \prime} & 1 & \tau \omega^{\prime} \\
\eta & \omega & \tau \omega^{\prime} \\
\eta \omega^{\prime} & \omega^{\prime} & \tau \omega \omega^{\prime \prime}
\end{array}\right] \\
& u_{\mathrm{f}}=\left[\begin{array}{lll}
\eta & 1 & \tau \\
\eta & 1 & 0 \\
\eta & 0 & \tau
\end{array}\right]
\end{aligned}
$$

where the statistical weight $\eta$ is the first-order interaction between $\mathrm{C}^{\alpha} \mathrm{H}$ and $\mathrm{C}^{\neq} \mathrm{H}, \tau$ is that between $\mathrm{C}^{\alpha} \mathrm{H}$ and $\mathrm{C}^{\ddagger} \mathrm{H}, \mathrm{CH}_{2} ; \omega, \omega^{\prime}$ and $\omega^{\prime \prime}$ are the second-order interaction between $\mathrm{CH}_{2}$ and $\mathrm{CH}_{2}, \mathrm{C}_{2} \mathrm{H}_{3}$ and $\mathrm{CH}_{2}, \mathrm{C}_{2} \mathrm{H}_{3}$ and $\mathrm{C}_{2} \mathrm{H}_{3}$, respectively. The statistical weight $\xi$ corresponding to the temperature $T$ may be defined by the relationship

$$
\begin{aligned}
& \xi=\xi_{0} \exp \left(-E_{\xi} / R T\right) \\
& \left(\xi=\eta, \tau, \omega, \omega^{\prime}, \omega^{\prime \prime}\right)
\end{aligned}
$$

where

$$
E_{\xi}=\left(E_{0} / 2\right)\left(1-\cos 3 \Phi_{j}\right)+\sum_{\alpha<\beta}\left(a_{\alpha \beta} / r_{\alpha \beta}^{12}-b_{\alpha \beta} / r_{\alpha \beta}^{6}\right)
$$

the barrier height $E_{0}$ is $11.70 \mathrm{kcal} \mathrm{mol}^{-1}$. The interaction parameters $a_{\alpha \beta}$ and $b_{\alpha \beta}$ are listed in Table I(a). The conformational energy $E_{\xi}$ and the conformational parameter $\xi_{0}$ are given by Table I(b). ${ }^{15}$ The geometric parameters for isotactic 1,2-PBD are listed Table I(c), and the dihedral angle between the plan of side groups and the plan of skeletal bonds in all-trans is $90^{\circ}$. Thus, $\Phi_{\mathrm{b}}^{\prime}$ can be calculated by

Table I(a). Exclusionary parameter $a_{\alpha, \beta}$ and London astigmatic parameter $b_{\alpha \beta}$

\begin{tabular}{ccc}
$\begin{array}{c}\text { Atom and } \\
\text { atoms pair }\end{array}$ & $\begin{array}{c}a_{\alpha \beta} \times 10^{-5} / \\
\AA^{12} \cdot \mathrm{kJ} \cdot \mathrm{mol}^{-1}\end{array}$ & $\begin{array}{c}b_{\alpha \beta} \times 10^{-2} / \\
\AA^{6} \cdot \mathrm{kJ} \cdot \mathrm{mol}^{-1}\end{array}$ \\
\hline $\mathrm{C} \cdots \mathrm{C}$ & 16.5 & 15.2 \\
$\mathrm{C} \cdots \mathrm{H}$ & 2.3 & 5.3 \\
$\mathrm{H} \cdots \mathrm{H}$ & 0.3 & 2.0 \\
$\mathrm{C}^{\neq} \cdots \mathrm{C} \neq$ & 46.7 & 22.8 \\
$\mathrm{C}^{\neq} \cdots \mathrm{C}$ & 27.9 & 18.6 \\
$\mathrm{C}^{\neq} \cdots \mathrm{H}$ & 4.3 & 6.5 \\
$\mathrm{C}_{2} \mathrm{H}_{3} \cdots \mathrm{C} \neq$ & 697.2 & 192.2 \\
$\mathrm{C}_{2} \mathrm{H}_{3} \cdots \mathrm{C}$ & 110.0 & 53.7 \\
$\mathrm{C}_{2} \mathrm{H}_{3} \cdots \mathrm{H}$ & 17.7 & 19.3 \\
\hline
\end{tabular}

Table I(b). The conformational energies $E_{\xi}$ and the conformational parameter $\xi_{0}$ for isotactic 1,2-PBD

\begin{tabular}{lcc}
\hline$\xi$ & $\xi_{0}$ & $E_{\xi} / \mathrm{J} \cdot \mathrm{mol}^{-1}$ \\
\hline$\eta$ & 1.0 & 1670 \\
$\tau$ & 0.4 & 4180 \\
$\omega$ & 0.7 & 2930 \\
$\omega^{\prime}$ & 1.2 & 5850 \\
$\omega^{\prime \prime}$ & 1.0 & 10450
\end{tabular}


Table I(c). The geometric parameter for isotactic 1,2-PBD

\begin{tabular}{lcll}
\hline Bond & $\begin{array}{c}\text { Bond } \\
\text { length } / \AA\end{array}$ & Bond angle & Angle $/^{\circ}$ \\
\hline $\mathrm{C}-\mathrm{C}^{\alpha}$ & 1.53 & $\angle \mathrm{CC}^{\alpha} \mathrm{C}^{\ddagger} \angle \mathrm{CC}^{\alpha} \mathrm{C}$ & 112 \\
$\mathrm{C}^{\alpha}-\mathrm{C}^{\ddagger}$ & 1.51 & $\angle \mathrm{C}^{\alpha} \mathrm{CC}^{\alpha}$ & 112 \\
$\mathrm{C}^{\ddagger}=\mathrm{C}$ & 1.35 & $\angle \mathrm{C}^{\alpha} \mathrm{C}^{\ddagger} \mathrm{C}$ & 120 \\
\hline
\end{tabular}

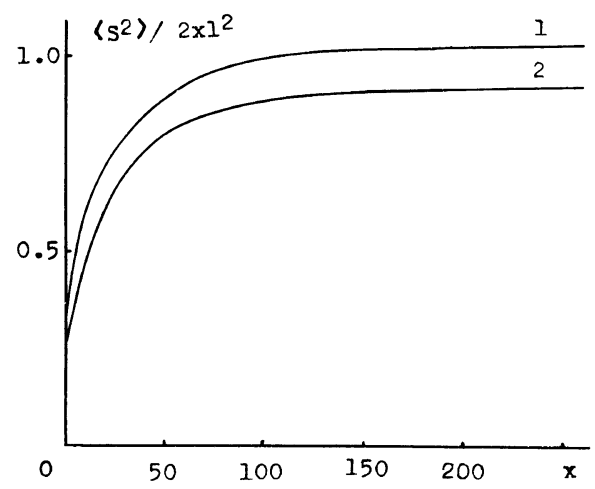

Figure 4. The characteristic ratios $\left\langle S^{2}\right\rangle / 2 x l^{2} v s$. the degree of polymerization $x$ at $340 \mathrm{~K}$ for isotactic 1,2 polybutadiene considering side groups 1 and without considering side groups 2 .

$$
\sin \Phi_{\mathbf{b}}^{\prime}=-\left(\cos \left(\theta_{\mathbf{a}} / 2\right)\right) / \sin \theta_{\mathbf{a}}^{\prime}
$$

Substituting the geometric parameters into eq $17-19$ and eq 63 , we have

$$
\left.\begin{array}{ll}
\Phi_{\mathrm{a}}^{\prime}=126.8^{\circ}, & \Phi_{\mathrm{f}}^{\prime}=233.2^{\circ} \\
\Phi_{\mathrm{b}}^{\prime}=-63.4^{\circ}, & \Phi_{\mathrm{e}}^{\prime}=73.2^{\circ}
\end{array}\right\}
$$

The characteristic ratios of mean-square radius of gyration $\left\langle S^{2}\right\rangle / 2 x l^{2} v s$. the degree of polymerization $x$ at $340 \mathrm{~K}$ for isotactic 1,2-PBD with considering side groups and without considering side groups may be numerical calculated shown in Figure 4, where $l$ is the length of bond $\mathrm{C}^{\alpha}-\mathrm{C}$. The ratio of mean-square radius of gyration for isotactic 1,2-PBD with considering side groups is greater $11 \%$ than that without considering side groups when $x$ exceeds 200. The root-mean-square radius of gyration, depended on the molecular weight $M$, is

$$
\left\langle S^{2}\right\rangle^{1 / 2}=0.298 M^{1 / 2}
$$

The mean-square radius of gyration for isotactic PP with considering side groups, which has been reduced as eq $14-47$, is given the identical result with the experimental data, i.e., the molecular weight dependence of rootmean-square radius of gyration is obtained as

$$
\left\langle S^{2}\right\rangle^{1 / 2}=0.34 M^{1 / 2}
$$

Besides, the relation for $\mathrm{PE}$ without considering side groups is given ${ }^{1}$

$$
\left\langle S^{2}\right\rangle^{1 / 2}=0.423 M^{1 / 2}
$$

and with considering side groups given ${ }^{10}$

$$
\left\langle S^{2}\right\rangle^{1 / 2}=0.44 M^{1 / 2}
$$

the latter is more approached to the experimental data ${ }^{9}$

$$
\left\langle S^{2}\right\rangle^{1 / 2}=0.45 M^{1 / 2}
$$

The ratio for $\mathrm{PE}$ with considering the side group is greater $8 \%$ than that without considering the side group. Obviously, if the geometric structure of side groups is considered, the centers of mass of monomers will be changed. Then, the distances of the center of mass of chain from the centers of mass of each monomer are changed. That is, the dimension of the polymer chain is also changed. Therefore, it is possible that the ratio of mean-square radius of gyration of polymer chains with considering the geometric structure of side groups is different form that without considering the geometric structure of side groups.

\section{REFERENCES}

1. P. J. Flory, "Statistical Mechanics of Chain Molecules," Interscience, New York, N.Y., 1969.

2. P. J. Flory, Macromolecules, 7, 381 (1974).

3. D. G. H. Ballard, J. Schelten, and G. D. Wignall, Eur. Polym. J.," 9, 965 (1973).

4. G. D. Wignall, D. G. H. Ballard, and J. Schelten, Eur. Polym. J., 10, 961 (1974).

5. J. Schelten, G. D. Wignall, and D. G. H. Ballard, Polymer, 15, 682 (1974).

6. K. Venkataswamy, A. M. Jamieson, and R. G. Petschek, Macromolecules, 19, 124 (1986). 
7. Y. Tamai, T. Konishi, Y. Einaga, M. Fujii, and H. Yamakawa, Macromolecules, 23, 4067 (1990).

8. Z. Zhiping, X. Jianmin, S. Xubing, and Y. Deyue, Eur. Polym. J., 28, 1339 (1992).

9. A. Zirkel, V. Urdan, and D. Richter, Macromolecules, 25, 6148 (1992).

10. Z. Zhiping, Y. Deyue, and T. Aoqing, Acta Chemica Sinica (Chinese) 50, 313 (1992).

11. P. Corradini, R. Napolitano, V. Petraccone, B. Pirozzi, and A. Tuzi, Macromolecules, 15, 1207 (1982).
12. C. D. Rosa, G. Zhi, R. Napolitano, and B. Pirozzi, Macromolecules, 18, 2328 (1985).

13. P. J. Flory, P. R. Sundararajan, and L. C. Debolt, J. Am. Chem. Soc., 96, 5015 (1974).

14. J. Ketelnar, "Chemical Constitution," Elsevier, New York, N.Y., 1958.

15. Z. Zinan and F. Zhiliu, Acta Chemica Sinica, (Chinese), 46, 165 (1988).

16. H. Markovitz, J. Chem. Phys., 20, 868 (1952).

17. J. E. Mark, J. Am. Chem. Soc., 88, 4354 (1966). 